\title{
Acute necrotising encephalopathy of childhood: a new syndrome presenting with multifocal, symmetric brain lesions
}

\author{
M Mizuguchi, J Abe, K Mikkaichi, S Noma, K Yoshida, T Yamanaka, S Kamoshita
}

Department of Mental Retardation and Birth Defect Research, National Institute of Neuroscience,

Kodaira, and

Departments of

Paediatrics, Tokyo

Metropolitan Fuchu

Hospital and

Neurological Hospital,

Fuchu, Japan

M Mizuguchi

Sendai Municipal

Hospital, Sendai,

Japan

$J$ Abe

Ohme Municipal

General Hospital,

Ohme, Japan

K Mikkaichi

Tokyo Metropolitan

Hachioji Children's

Hospital, Hachioji,

Japan

S Noma

Matsuyama Shimin

Hospital, Matsuyama,

Japan

K Yoshida

Yaizu Municipal

Hospital, Yaizu, Japan

$T$ Yamanaka

University of Tokyo,

Japan

S Kamoshita

Correspondence to:

Dr M Mizuguchi,

Department of Mental

Retardation and Birth Defect

Research, National Institute

of Neuroscience, NCNP,

of Neuroscience, NCNP,

Kodaira, 187, Japan

Received 22 July 1994

and in revised form

10 November 1994.

Accepted 22 December 1994

\begin{abstract}
The clinicopathological features of a previously unrecognised type of acute encephalopathy prevalent among Japanese children is described by reviewing the records of 13 consecutive patients treated and 28 previously reported cases. The hallmark of this encephalopathy, proposed to be a novel entity termed acute necrotising encephalopathy of childhood, is multiple, necrotic brain lesions showing a symmetric distribution. The encephalopathy was noted in previously healthy children after respiratory tract infections, with presenting symptoms of coma, convulsions, vomiting, hyperpyrexia, and hepatomegaly. Laboratory examinations disclosed liver dysfunction, uraemia, and hypoproteinaemia. The histological appearance of the liver was variable and non-specific. Cerebrospinal fluid contained an increased amount of protein. Computed tomography and MRI showed the presence of symmetrically distributed brain lesions of the thalamus, cerebral white matter, brainstem, and cerebellum. Necropsy examination confirmed extensive fresh necrosis of these regions with evidence of local breakdown of the blood-brain barrier. Based on the characteristic combination of clinical and pathological findings, acute necrotising encephalopathy of childhood can be distinguished from previously known encephalopathies, including Reye's syndrome.
\end{abstract}

\section{$(\mathcal{F}$ Neurol Neurosurg Psychiatry 1995;58:555-561)}

Keywords: acute encephalopathy; computed tomography; pathology

The introduction of CT into medical practice in the late 1970s in Japan has led to the recognition of cases of an acute encephalopathy showing unique brain pathology, which is characterised by the presence of multifocal brain lesions in the bilateral thalamus, brainstem, tegmentum, and cerebellum.

The first case was reported in 1979, diagnosed as Reye's syndrome with unusual CT findings. ${ }^{1}$ During 1983 to 1986 , five cases encountered at one of our hospitals were reported, together with the suggestion that the condition may constitute a novel subtype of acute encephalopathy. ${ }^{2-4}$ These reports may have prompted subsequent reports of at least 28 cases noted at other institutions in Japan, ${ }^{5-25}$ although the identity of the disease has so far remained obscure.

To facilitate data acquisition, our institutions have conducted a collaborative study. This report presents the results of that study and a survey of the clinical, radiological, and pathological features of the disorder conducted in an effort to elucidate its identity as a novel disease entity, which we have designated by the term acute necrotising encephalopathy of childhood (ANE).

\section{Patients and methods}

From among patients with acute encephalopathy in childhood, we retrospectively selected those who met the following criteria: acute non-inflammatory encephalopathy with alteration in level of consciousness (CSF leucocyte count $8 / \mathrm{mm}^{3}$ or less); demonstration by CT of multifocal lesions symmetrically distributed in brain regions including the thalamus; and the absence of any other reasonable explanation for the cerebral abnormalities. Of the 13 such patients for whom records were retained at our hospitals (group A), five have been reported previously. ${ }^{2-4}$ Twenty eight additional patients at other institutions for whom sufficient acute stage data were available in the medical literature (group B) were also reviewed. ${ }^{5-25}$ The data for both groups are described together and combined for the calculation of percentages because of the relatively small sample size and the similarity between the two groups.

\section{Results}

BACKGROUND OF PATIENTS

All of the patients were Japanese children living in the central districts of Japan. The disease affected young children of both sexes, and $46 \%$ of the patients were between 6 and 18 months of age (table 1). The onset of disease was most often in the winter $(51 \%$ in December-February). At four hospitals, successive admission of two to four patients was noted. ${ }^{101221}$ Two such clusters, one in Sendai in December, 1985, and the other on the outskirts of Tokyo in March-June, 1988, ${ }^{21}$ were associated with epidemics of influenza $A$.

The history was unremarkable in most of the patients, although a minority had mild growth and developmental retardation (16\%) or somatic anomalies (16\%), such as cleft palate with dysmorphic facies, ventricular 
Table 1 Clinical findings at the acute stage

\begin{tabular}{|c|c|c|c|}
\hline Findings & Group $A$ & Group B & $(\%)^{*}$ \\
\hline \multicolumn{4}{|l|}{ Age: } \\
\hline Mean (SD) (y) & $2 \cdot 0(1 \cdot 3)$ & $2.6(2 \cdot 5)$ & \\
\hline Range & 9 months to & 5 months to & \\
\hline \multirow{2}{*}{\multicolumn{4}{|c|}{ 然 }} \\
\hline Male & $6 / 13$ & & \\
\hline Female & $7 / 13$ & $13 / 26$ & (51) \\
\hline \multicolumn{4}{|l|}{ Prodromes: } \\
\hline Common cold & $13 / 13$ & $25 / 26$ & (97) \\
\hline Fever $\left(>37 \cdot 5^{\circ} \mathrm{C}\right)$ & $13 / 13$ & $16 / 20$ & (88) \\
\hline \multicolumn{4}{|l|}{ Symptoms and signs: } \\
\hline Coma & $13 / 13$ & $28 / 28$ & $(100)$ \\
\hline Convulsions & $13 / 13$ & $21 / 22$ & (97) \\
\hline Vomiting & $10 / 13$ & $9 / 15$ & (68) \\
\hline Hyperpyrexia & $10 / 12$ & $14 / 17$ & (83) \\
\hline Hyperventilation & $11 / 11$ & $8 / 10$ & (90) \\
\hline Shock & $5 / 13$ & $4 / 12$ & (36) \\
\hline Diarrhoea & $7 / 13$ & $3 / 9$ & (45) \\
\hline Hepatomegaly & $7 / 13$ & $4 / 11$ & (46) \\
\hline Jaundice & $0 / 13$ & $0 / 7$ & (0) \\
\hline \multicolumn{4}{|l|}{ Decerebrate or decorticate } \\
\hline posture & $10 / 13$ & $17 / 18$ & (87) \\
\hline Miosis & $11 / 11$ & $7 / 12$ & (78) \\
\hline Sluggish light reflex & $1 / 11$ & $4 / 12$ & (22) \\
\hline Papilloedema & $2 / 5$ & $3 / 8$ & (38) \\
\hline Exaggerated tendon jerks & $6 / 10$ & $10 / 14$ & (67) \\
\hline Positive Babinski's sign & $4 / 10$ & $7 / 11$ & (52) \\
\hline
\end{tabular}

septal defect with bilateral radial agenesis and unilateral renal hypoplasia, retention testis, and polydactyly. ${ }^{10}$ No patient had travelled abroad. All of the cases were sporadic. The family history was negative for consanguinity and neurological disorders, except in one patient who had three paternal aunts who had died of Ekiri, a fulminant form of acute encephalopathy secondary to Shigella dysenteriae infection, which has now disappeared but was prevalent in Japan until the 1950s.

\section{SIGNS AND SYMPTOMS}

The onset of the encephalopathy was preceded by prodromal febrile illness with signs and symptoms of upper respiratory infection (table 1). Skin rash was noted in only one patient (exanthem subitum). ${ }^{9}$ Antipyretics $(61 \%)$, antibiotics $(23 \%)$, and other drugs were prescribed, but $17 \%$ of the patients received no medication. Aspirin had been taken by only two patients. The possibility of ingestion or inhalation of toxic substances, as well as attempted treatment with home remedies, was denied by the parents. After an interval of 0.5 to three days, without recovery from the prodrome, the initial signs and symptoms of brain dysfunction developed (table 1); these included impairment of consciousness, convulsions, and recurrent vomiting. The level of consciousness deteriorated rapidly, with development of coma within 24 hours, although one patient showed a somewhat protracted course. ${ }^{13}$ The types of convulsions were variable, but generalised tonic or tonic-clonic seizure were the most common ( $84 \%)$. The vomitus was bloody in $32 \%$ of the patients, but no haemorrhage at other sites was noted. Half of the patients had mild diarrhoea. Hepatomegaly $(\geqslant 2 \mathrm{~cm}$ below the costal margin) was noted either initially or later in the course, but was unaccompanied by visible jaundice. At the stage of coma, hyperpyrexia, hyperventilation, and decorticate or decerebrate posture were common findings. The pupils showed miosis, but were isocoric and promptly reactive to light in $78 \%$ of the patients. Mild papilloedema was present in a third of the patients. Deep tendon reflexes were mildly exaggerated and Babinski's sign was present bilaterally. Meningeal signs, abnormal involuntary movements, and focal neurological signs were absent, although in $23 \%$ of the patient's motor signs were more accentuated on one side than on the other.

The disease reached its peak in the first few days. High fever lasted for two to five days. Systemic hypotension developed in the severe cases. ${ }^{6} 1318$ Despite treatment, death occurred in $28 \%$ of the patients. In the surviving patients, the recovery of consciousness was first noted on the sixth to the 10th day. Although improvement of neural functions continued for several months, most patients had residual neurological sequelae, such as spastic tetraplegia with rigidity $(63 \%$ of the survivors), severe mental delay (63\%), and epileptic seizures (24\%). ${ }^{57}$ In milder cases, there were focal signs such as hemiparesis (four patients), ${ }^{11} 22$ slurred or scanning speech, intention tremor, or ataxia, or both (four patients), ${ }^{19} 20$ and abducens nerve palsy (one patient). ${ }^{19}$ Compared with the motor signs, cognitive functions showed good recovery. ${ }^{13192122}$ Recurrence of acute episodes or subsequent further deterioration was not encountered during the follow up period.

\section{LABORATORY FINDINGS}

Biochemical examinations of blood on admission showed increases in serum aspartate aminotransferase (95\%, range 56-11 480 IU/1), alanine aminotransferase ( $81 \%$, range 10-12 $300 \mathrm{IU} / 1)$, lactate dehydrogenase $(87 \%)$, creatine kinase $(48 \%)$, and blood urea nitrogen $(90 \%)$, but not in creatinine $(16 \%)$. Increased blood ammonia $(6 \%)$ and total bilirubin (13\%) were rare. The serum calcium concentration was slightly depressed (50\%), and sodium was in the low normal range. There was no hypoglycaemia (3\%). Metabolic acidosis of variable degree was present $(88 \%)$. Increases in blood concentrations of lactate $(19 \%$; range $4.8-19.2 \mathrm{mg} / \mathrm{dl})$ and pyruvate (38\%; range $0.4-1.51 \mathrm{mg} / \mathrm{dl}$ ) were occasional and slight.

During the first two days after admission, the biochemical abnormalities progressed. In $55 \%$ of the patients, hypoproteinaemia developed during this period, ${ }^{10}$ to as low as $3.6 \mathrm{~g} / \mathrm{dl}$ in the most severe case. In the surviving patients, the first evidence of normalisation of laboratory values was seen on the third to sixth day, and complete normalisation within three to five weeks.

Haematological examinations showed no rapid decrease in haemoglobin concentration $(0 \%)$. In the acute period, low platelet count $(50 \%)$, prolonged prothrombin time or partial thromboplastin time (38\%), decreased fibrinogen $(54 \%)$, and increased fibrin degradation products $(57 \%)$ were detected in severe cases, although not to the degree that they fulfilled the criteria for disseminated intravascular coagulation. Serological examination 
Table 2 Computed tomography findings

\begin{tabular}{|c|c|c|c|}
\hline Findings & Group $A$ & Group B & $(\%)^{*}$ \\
\hline \multicolumn{4}{|l|}{ Brain contour on initial CT: } \\
\hline Oedema & $9 / 13$ & $16 / 21$ & (74) \\
\hline Atrophy & $1 / 3$ & $1 / 21$ & (6) \\
\hline \multicolumn{4}{|l|}{ Distribution of lesions: } \\
\hline Thalamus and internal capsule $\dagger$ & $13 / 13$ & $28 / 28$ & $(100)$ \\
\hline Cerebral white matter + & $8 / 13$ & $15 / 21$ & (68) \\
\hline Cerebellum§ & $11 / 13$ & $13 / 21$ & $(71)$ \\
\hline Pons and/or midbrain $\|$ & $12 / 13$ & $12 / 20$ & (73) \\
\hline Other brain regions & $0 / 13$ & $0 / 28$ & (0) \\
\hline \multicolumn{4}{|l|}{ Residual lesions: } \\
\hline Cerebral atrophy & $6 / 7$ & $9 / 13$ & (75) \\
\hline Small thalamic hypodensity & $4 / 7$ & $12 / 16$ & (70) \\
\hline Multiple white matter cysts & $4 / 7$ & $4 / 12$ & (42) \\
\hline
\end{tabular}

^Total for groups A and B; †posterior limb; †periventricular region; §medullary substance around the dentate nucleus; $\|$ tegmentum.
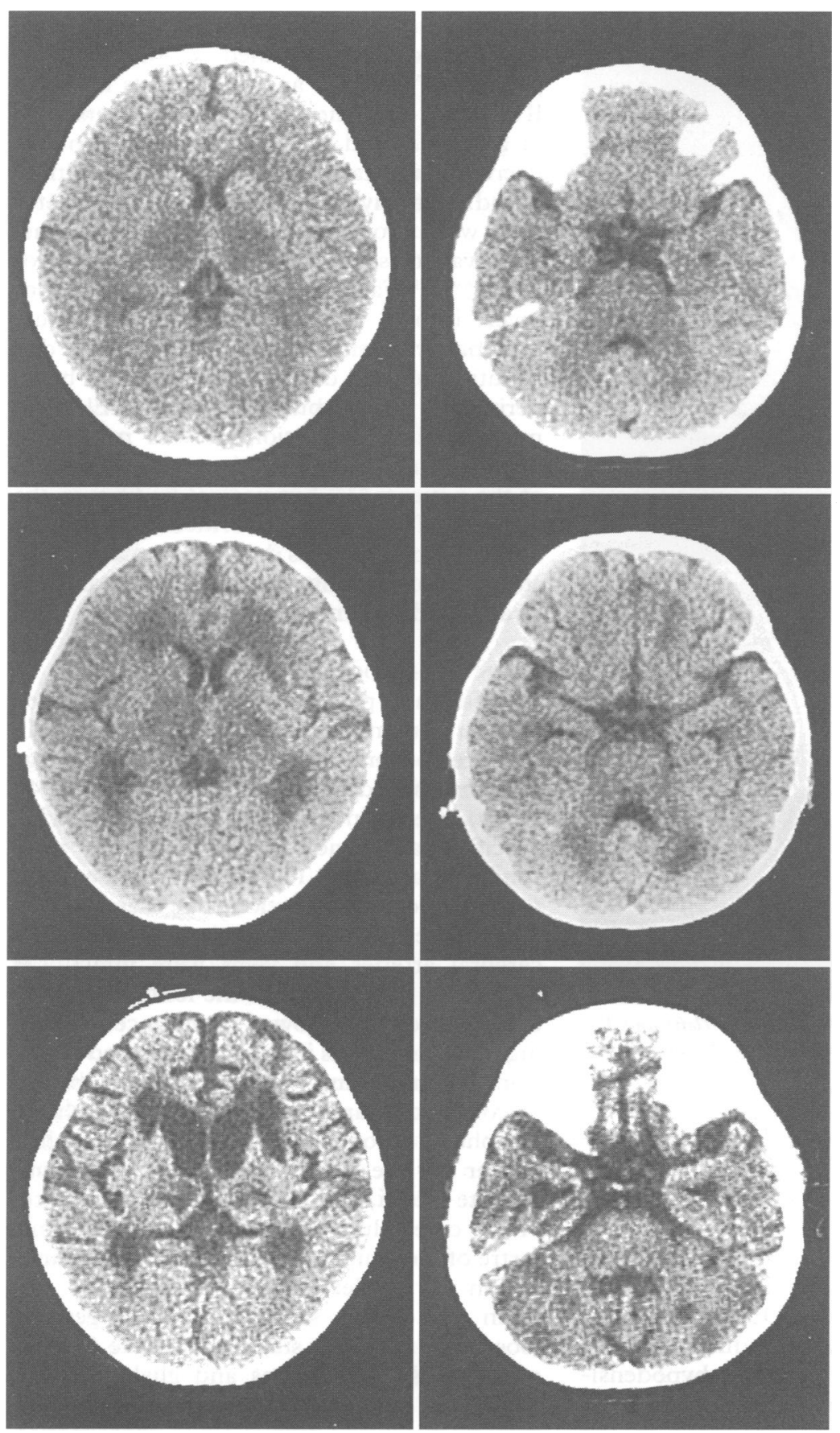

Figure 1 CT findings in a female patient, 1 year and 3 months of age. On the second day of illness (top), multiple, symmetric hypodense areas in the thalamus, cerebellum, and brainstem are already apparent. The periventricular white matter shows slight reduction of attenuation. On the eighth day (middle), the density of the lesions is further decreased, and the thalamic lesions contain tiny hyperdense dots. The cerebrum is slightly atrophic. On the 41 st day (bottom), the hypodense areas are reduced in size, but remain as cystic lesions. The cerebrum shows severe atrophy. showed positive $\mathrm{C}$ reactive protein $(76 \%)$ and a modest increase of erythrocyte sedimentation rate $(42 \%)$. During their stay in hospital significant increases in antibody titres for influenza $\mathrm{A}^{121}$ (five patients; 24\%) and coxackie A9 (one patient) was detected. Rotavirus was isolated from the stool of two patients, and influenza B from the pharynx of two patients. ${ }^{6}$ Polymerase chain reaction analysis on the CSF of five patients failed to detect herpes virus DNA. Bacteriological studies of blood, CSF, and stool specimens yielded negative results.

The findings for urine were unremarkable except for transient proteinuria $(50 \%)$ and haematuria $(33 \%)$. Serum and urine analysis of amino acids and organic acids disclosed no specific abnormalities (both $0 \%) .{ }^{71014}$ Serum carnitine and urine acylcarnitine concentrations were normal (two patients).

Spinal tap disclosed high CSF pressure. Concentrations of protein (78\%) and myelin basic protein (83\%) in CSF were increased, ${ }^{202125}$ whereas CSF lactate and pyruvate concentrations were normal $(0 \%$ and $17 \%$ respectively). ${ }^{7125}$ During convalescence, all the CSF findings returned to normal.

\section{ELECTROPHYSIOLOGICAL FINDINGS}

The EEG at the acute stage was dominated by diffuse $1-6 \mathrm{~Hz}$ slow waves except in brain dead patients (100\%). Paroxysmal activities were rare in the initial record $(18 \%),{ }^{13}{ }^{14}$ but were common in the follow up EEG several weeks later. The auditory brainstem response was diminished or abolished $(86 \%) .^{718}$

\section{NEURORADIOLOGICAL FINDINGS}

In all the patients, CT showed the presence of multiple low density areas in the brain (fig 1). The lesions were not seen in the two patients examined early in the lethargic state, ${ }^{1}$ but were apparent within 12 hours after the onset of coma (100\%). The brain was oedematous in most patients, but mild atrophy was noted in a few (table 2). The distribution of the hypodense lesions was almost symmetric and was similar in all the patients (table 2). Supratentorial lesions included most of the thalamus, the posterior limb of the internal capsule, and the posterior part of the lenticular nucleus. The periventricular white matter of the cerebrum was also affected. Infratentorial lesions were distributed in the cerebellar medullary substance around the dentate nucleus and the tegmentum of the pons or midbrain. Other brain regions were not affected in any patient. The thalamic lesions were enhanced by contrast materials in $27 \%$ of the patients.

During the first week of illness, the thalamic lesions became mottled, and the cerebral white matter lesions became more hypodense. During subsequent weeks, the thalamic and infratentorial lesions became smaller and the cerebrum more atrophic. Five patients in group B showed positive contrast enhancement at this stage, notably ring like enhancement of the margin of the thalamic 


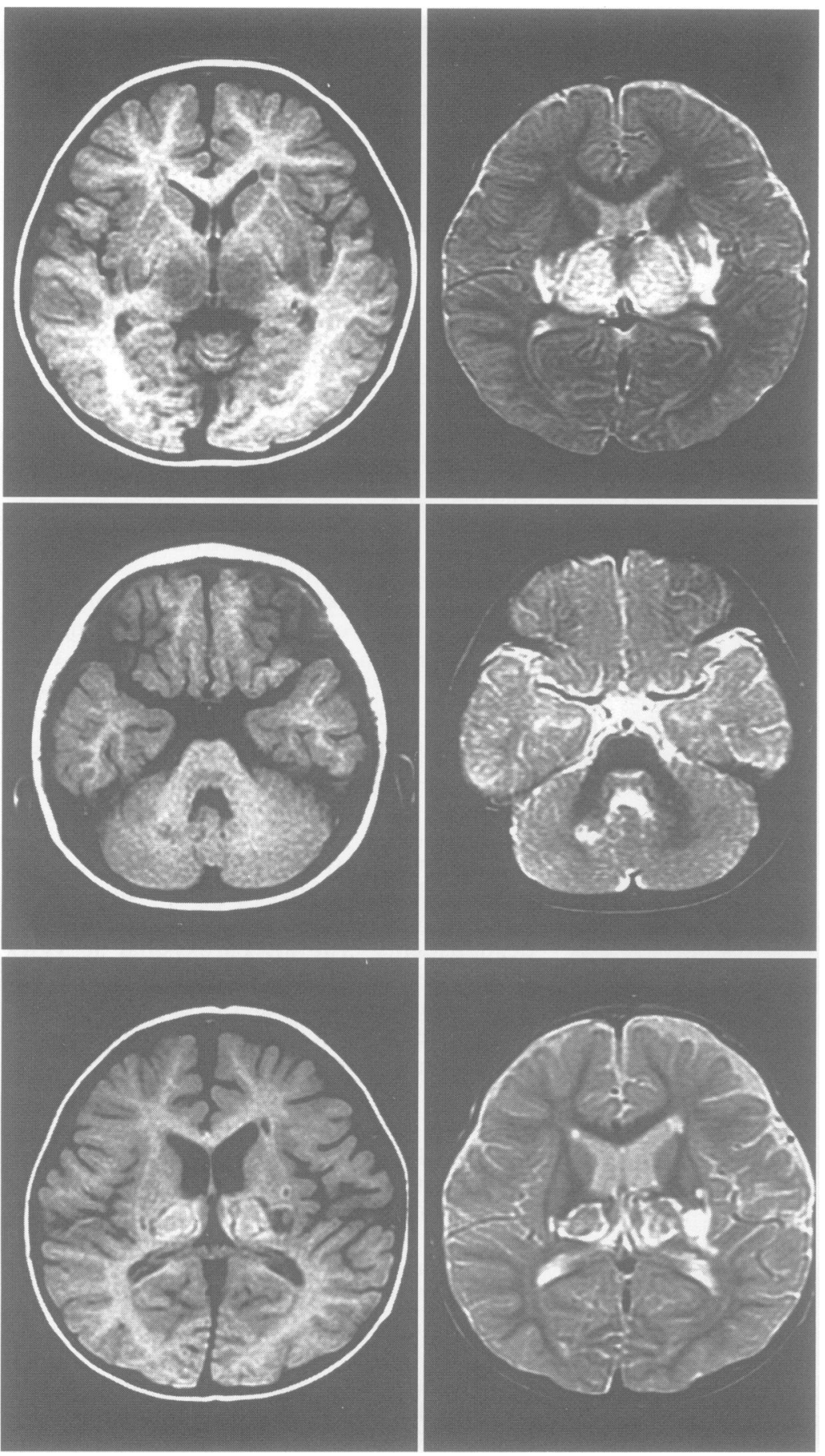

Figure 2 MRI findings in a male patient, 1 year and 3 months of age. T1 (left) and T2 (right) weighted images on the fourth day (top and middle) and 23rd day (bottom) of illness show symmetric lesions in the thalamus, internal capsule, lenticular nucleus, periventricular white matter, cerebellum, and pons. The infratentorial and cerebral white matter lesions in this patient are small. At the initial MRI (fourth day), the signal intensity observed for the lesions is low on $T 1$ weighted images and high on $T 2$ weighted images. At the subsequent MRI (23rd day), the centre and periphery of the thalamic and lenticular lesions show hyperintensity on both $T 1$ and $T 2$ weighted images, suggestive of haemorrhagic change. lesions was found on T1 weighted images and high intensity on T2 weighted images, the thalamic lesions in all patients included areas of high signal intensity on both $\mathrm{T} 1$ and $\mathrm{T} 2$ weighted images (fig 2). In two patients, the high intensity had consisted solely of minute dots during the initial two to four days but became larger and coalescent during subsequent weeks. ${ }^{23}$ In the other patients, an oval area of high intensity in the centre and a ring like zone of high intensity along the margin were prominent as early as the first week of illness. ${ }^{19-21}$ In no patient was intravascular thrombosis demonstrated.

\section{LIVER PATHOLOGY}

Histological examinations were performed on tissue specimens obtained at biopsy (four patients in group. A and 10 patients in group B) ${ }^{917-192123}$ or at necropsy (two patients each in groups $\mathrm{A}$ and B). ${ }^{617}$ In no specimen was necrosis, bile stasis, or inflammatory infiltrate found. In many of these patients, the hepatocytes were swollen or contained intracytoplasmic vacuoles that stained positively for lipids. ${ }^{691821}$ Fatty changes were moderate or severe in $61 \%$ of the patients, ${ }^{691821}$ but were minimal or absent in the others. ${ }^{142123}$ The distribution of lipid droplets was variable, being periportal, centrilobular, or diffuse. Electron microscopic examination showed mitochondrial swelling and mild pleiomorphism in $58 \%$ of the patients, ${ }^{621}$ but normal findings in the others. ${ }^{18}$ There was no evidence of further abnormalities, such as decrease in matrix density, loss of intramitochondrial dense bodies, or ameboid deformation.

\section{BRAIN PATHOLOGY}

Permission to perform necropsy was obtained for two patients each in groups A and B. ${ }^{6}$ In the three patients who were in the acute stage at necropsy (duration of illness six to 48 hours), the brain was the only organ showing specific pathological changes, which were similar in all three. The brains were oedematous on gross examination, but there was no cerebral herniation. The main branches of the cerebral arteries and veins were intact. On sectioning (fig $3 \mathrm{~A}$ ), all of the lesions that had been seen on CT showed pronounced softening. Central lesions in the thalamus and brainstem tegmentum showed dark brown discoloration and petechial haemorrhage. Peripheral lesions in the periventricular white matter of the cerebrum, corpus callosum, dentate nuclei, and surrounding white matter of the cerebellum showed loss of the normal lustre of the white matter. Microscopic examination of the lesions by haematoxylin and eosin and myelin stains showed congestion, loosening, and poor staining of tissue (fig 3B) and necrosis of neurons and glial cells, but there was no proliferation of reactive astrocytes or microglial cells. A silver impregnation method showed concomitant loss of axons. Around the small arteries, veins, and capillaries, extravasation of erythrocytes was seen in the central lesions (fig 3C) and a plasma like substance was present in the peripheral 
Figure 3 Necropsy findings for the brain of a male patient, 1 year and 9 months of age, who died on the first day of illness. (A) Gross appearance of the unfixed brain section. The brain is oedematous; the thalamus and lenticular nucleus are softened and discoloured dark brown; and the periventricular white matter and corpus callosum are also sofiened. (B) $A$ periventricular lesion, abutting the cingulate gyrus (right upper) and involving part of the corpus callosum (right lower), is demonstrated as a sharply demarcated area of myelin pallor (luxol fast blue). (C) In the thalamic lesion there are: loosening of the tissue, petechial haemorrhage, and scattered cells with darkly stained nuclei. (D) In the cerebral white matter lesion, extravasation of a plasma like substance is noted around a vein (C and $D$, haematoxylin and eosin).

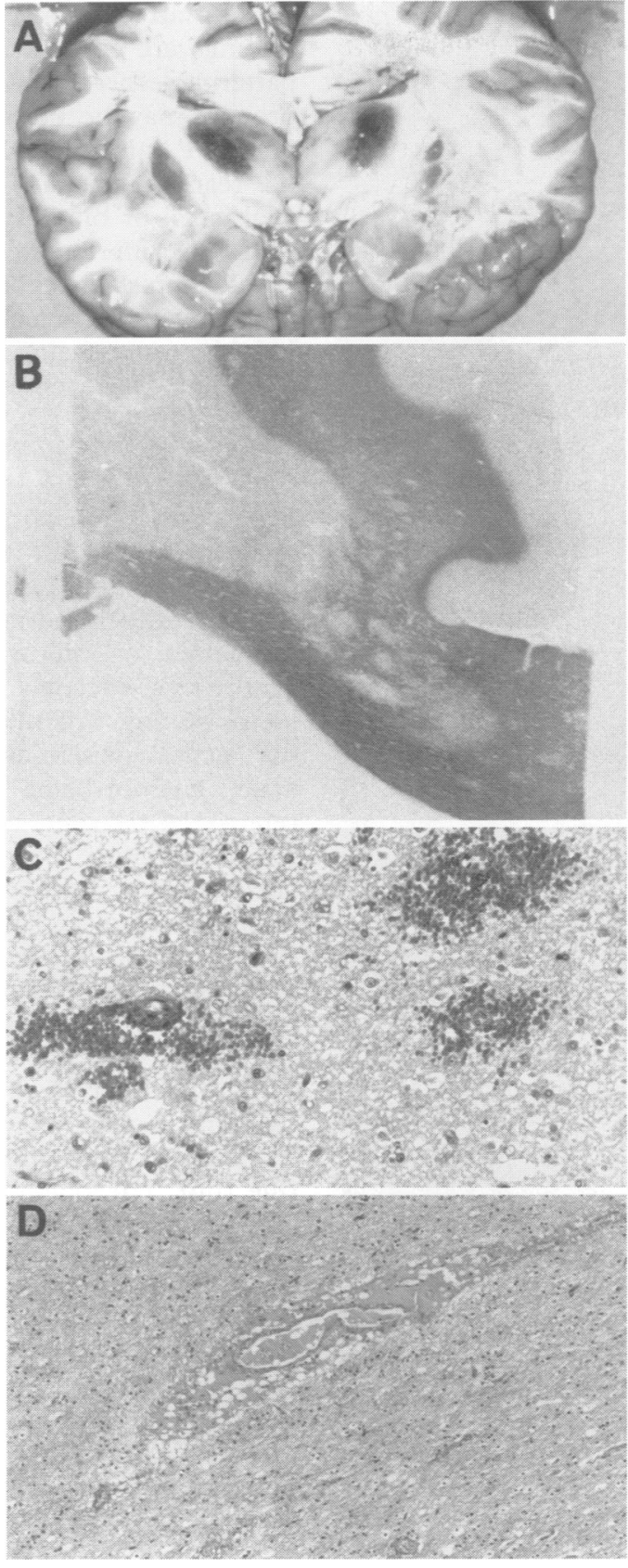

lesions (fig 3D). Except for occasional nuclear pyknosis of the endothelial cells, no notable change of blood vessels, such as deposition of fibrinoid materials or infiltration of inflammatory cells in the perivascular space, was noted. The walls of arteries and veins appeared intact by elastic-van Gieson and connective tissue stains, and the vascularity was normal by reticulin stains.

The brain of a patient who died after one year of persistent vegetative state $^{14}$ was atrophic with enlargement of the ventricles. The thalami and basal ganglia showed softening, microcavitation, or brown discoloration. There were multiple cystic lesions in the periventricular white matter of the cerebrum (fig 4A). In the brainstem of this patient, considerable atrophy of the tegmentum was present at both the mesencephalic and pontine levels. There were bilateral symmetric, necrotic lesions in the pontine tegmentum and cerebellar medullary substance, and a cystic lesion in the midbrain (fig 4B). On histological examination, the dorsal half of the thalamus and adjacent corpus striatum showed multiple necrotic foci filled with lipid laden macrophages (fig 4C). In the other lesions of the grey matter, such as the ventral half of the thalamus, severe loss of neurons and myelin sheaths were present together with gliosis. Fibrous gliosis was also noted along the walls of cavities in the white matter and the midbrain.

At both the acute and chronic stages, the boundaries of the lesions were sharply defined but were irrespective of those of normal structures. Outside the affected areas, there were no abnormal findings except for mild ischaemic change in neurons.

\section{Discussion}

The clinical, radiological, and pathological features of the encephalopathy described in this report constitute an unusual but characteristic pattern that we believe warrants recognition of ANE as a distinct clinicopathological entity. Although the individual clinical or laboratory findings in ANE may be seen in a wide range of disorders, including overwhelming bacterial and viral infections, fulminant hepatitis, toxic shock, haemolyticuraemic syndrome, other toxin induced diseases, Reye's syndrome, haemorrhagic shock and encephalopathy syndrome, and heatstroke, none of these conditions are associated with the symmetric brain lesions seen in ANE. Multifocal haemorrhagic or nonhaemorrhagic infarcts have been found in the brains of patients with haemolytic-uraemic syndrome and haemorrhagic shock and encephalopathy syndrome, but they are usually asymmetric. ${ }^{26}{ }^{27}$ Moreover, the cardinal clinical and laboratory findings in ANE are clearly distinct from the features of these diseases, with the sole exception of Reye's syndrome.

The differentiation of ANE from Reye's syndrome has presented much difficulty in the patients who have very high transaminase values or fatty change of the liver, some of whom were initially thought to have Reye's syndrome. ${ }^{1468917182125}$ There are certain differences, however, between ANE and Reye's syndrome; diarrhoea and increased CSF protein are common in ANE but are rare in Reye's syndrome, patients with ANE do not present certain metabolic features of Reye's syndrome such as hyperammonaemia and hypoglycaemia, ${ }^{28}$ and, unlike Reye's syndrome, ANE is not associated with varicella as an antecedent infection. With regard to the liver pathology, the combination of encephalopathy and fatty liver change alone is not conclusive evidence warranting the diagnosis of Reye's syndrome. Fatty change in the liver has been regarded by some investigators as an incidental and non-specific finding in Reye like encephalopathies. ${ }^{29}$ This view seems to hold true in ANE, as the number and distribution of lipid droplets are highly variable among the patients. Moreover, the severe 


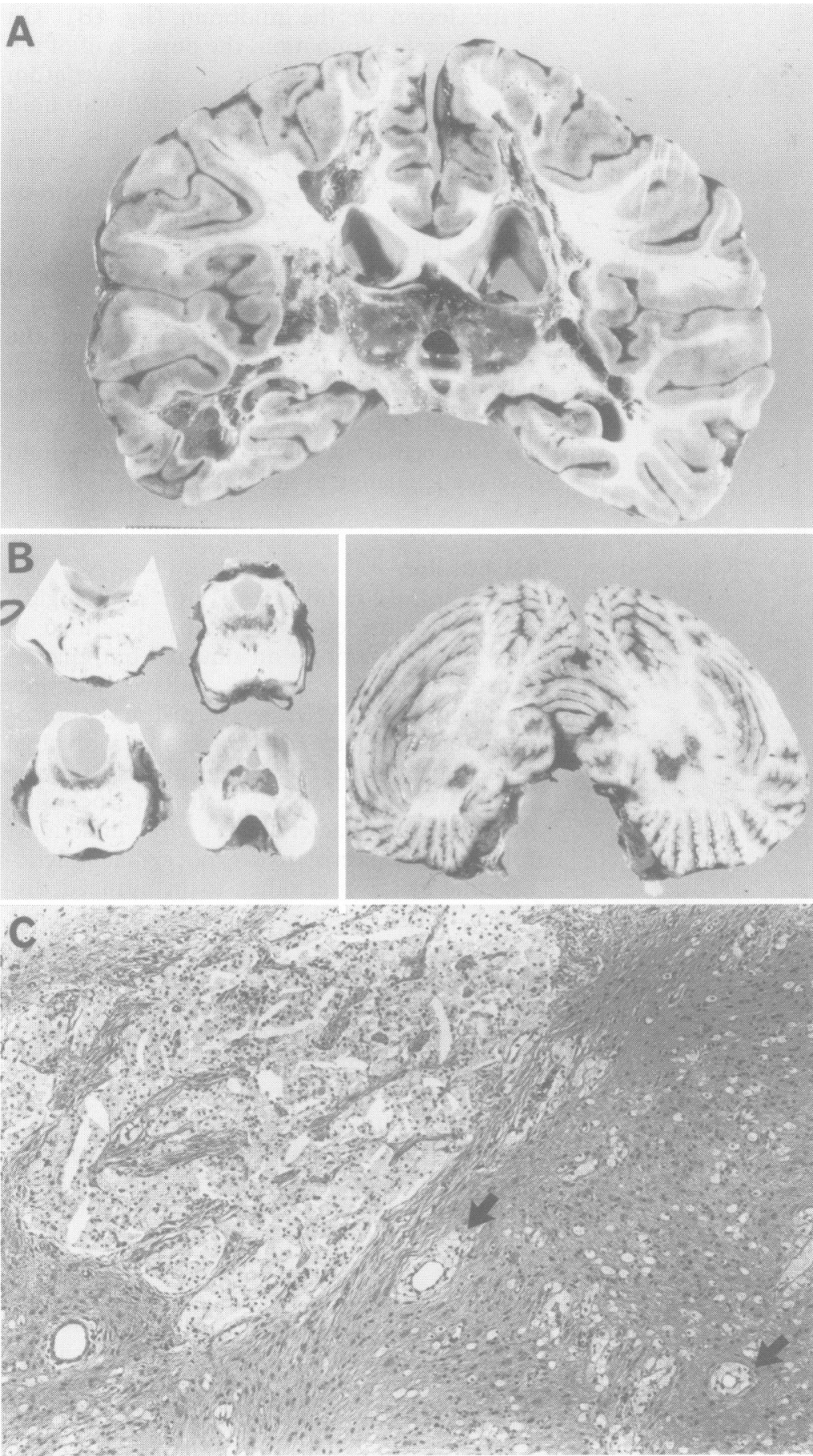

Figure 4 Gross necropsy findings for the brain of a female patient who had contracted encephalopathy at the age of 8 months, one year before her death. $(A)$ The cerebrum shows atrophy, pigmentation (brown) of the thalamus, and the presence of multiple cavities in the white matter surrounding the lateral ventricle. (B) The brainstem shows selective atrophy of the tegmentum, where cysts and necrotic (brown) lesions are present symmetrically. Symmetric lesions are also noted in the cerebellum. (C) In the thalamic lesion, there are macrophage filled, necrotic foci (left) and perivascular aggregates of macrophages (arrows). The rest of the tissue shows severe gliosis (haematoxylin and eosin). white matter lesions; both of these necropsy findings are compatible with the local signal patterns seen on MRI. The increase in CSF protein may be additional evidence for the defective barrier. At the acute stage, there are no reactive changes of inflammatory cells, glial cells, or blood vessels. Absence of such reactions differentiates ANE from Wernicke's and Leigh's encephalopathies, and results from the short clinical course of ANE. Unlike Leigh's encephalopathy, ANE shows a fulminant and monophasic course without exception.

From the radiological and pathological standpoints, ANE should be differentiated from other acute disorders that may produce bilateral lesions in the cerebral deep grey matter, including Leigh's encephalopathy and related mitochondrial cytopathies, glutaric acidaemia, methylmalonic acidaemia, Wernicke's encephalopathy, carbon monoxide poisoning, infantile bilateral striatal necrosis, acute disseminated encephalomyelitis, acute haemorrhagic leucoencephalitis (also referred to as "acute necrotising haemorrhagic encephalopathy"), and other types of encephalitis, vasculitis, arterial or venous infarction, and the effects of severe hypoxia or head trauma. Of these, the metabolic disorders can be excluded based on biochemical studies, and the intoxications based on clinical history. Infantile bilateral striatal necrosis shows a different distribution, the corpus striatum being affected most severely. ${ }^{31}{ }^{32}$ Although it may occasionally be difficult to rule out encephalitides solely on clinical grounds, the brain pathology in ANE is not compatible with inflammatory disorders. For example, the brain lesions in ANE lack perivascular and meningeal inflammation, necrosis of blood vessels, and asymmetric distribution, features that are typical of acute haemorrhagic leucoencephalitis. ${ }^{33}$

The brain lesions in ANE cannot be ascribed to incidental circulatory accidents such as hypoxia or ischaemia, as has been pointed out by some previous authors who made the diagnosis of Reye's syndrome, ${ }^{7-10}$ in which the gross neuropathological findings are essentially limited to brain oedema. ${ }^{34}$ Diffuse cerebral hypoxia can result in symmetric damage to the bilateral deep grey matter, but such a pathomechanism is excluded by the clinical course in many of the patients; the CT lesions appear early in the absence of hypoxic-ischaemic episodes. Local ischaemia due to hypoperfusion of the branches of the bilateral posterior cerebral arteries has been proposed, with speculated compression of the arteries caused by transtentorial herniation. ${ }^{735}$ This explanation, however, is refuted by the presence of lesions beyond the territory of the posterior cerebral artery, the absence of any sign of brain oedema on the initial CT scan in $26 \%$ of the patients, and the absence of cerebral herniation in the necropsied brains. Thrombotic occlusion of large cerebral arteries or veins is another possibility. In exceptional cases, in which the lesions were restricted to the thalamus and lenticular nucleus, it is possible that bilateral infarction of erythrocytes in the thalamic lesions and that of a plasma like substance in the cerebral 
developed due to thrombosis of the internal cerebral veins, great cerebral vein of Galen, or straight sinus. ${ }^{2436}$ In most of the cases, however, a thrombotic mechanism is not tenable because of the lack of CT or MRI evidence, the intact vessels found on postmortem examinations, and the more extensive distribution of lesions than the territories fed by a single artery or those drained by a single vein.

Although the basis for the distribution remains obscure, the affected areas generally correspond to the zones irrigated by the terminal branches of the intracerebral arteries and to the regions where $\mathrm{T} 1$ and $\mathrm{T} 2$ relaxation times on MRI decline early in normal development, ${ }^{37}$ implicating regional differences in blood flow and myelination as the factors responsible for the selective vulnerability.

Because biochemical studies have so far provided no promising clue as to the pathogenesis of ANE, further epidemiological investigations are needed. The geographical distribution of ANE is noteworthy. It is by no means uncommon among Japanese children, and there are reports of adult cases showing similar pathological findings, that may represent a variant of ANE. ${ }^{38}$ By contrast, we have so far failed to find any reports of similar cases occurring outside Japan. We hope that this report will serve to alert paediatric neurologists and neuropathologists in countries other than Japan, where there may be as yet unrecognised cases of ANE. Detailed epidemiological, clinical, and pathological investigations may shed light on the aetiology of this devastating disorder.

We thank Drs Rinmei Fukuda, Masaki Hoshiyama, Kunio Kaneshi, Hajime Katayama, Eiji Kurihara, Akira Kusakari, and Mineko Matsuo for clinical evaluations, Drs Nobuhik Aoki and Akira Yagishita for radiological interpretations, and Drs Koken Kinjo and Toshihiro Nishida for pathological investigations.

1 Mizuta R, Izumi H, Takeuchi S, et al. A case of Reye's syndrome with elevation of influenza A, CF antibody. syndrome with elevation of influenza A, CF antibody.
fapanese fournal of Pediatrics 1979;32:2144-9. (In fapanese
Japanese.)

2 Aoki N, Kaneshi K, Mizuguchi M, Kurihara E. Computed tomography in acute toxic encephalopathy-Report of three cases with symmetrical low density areas in the thalamus and the cerebellum. No To Hattats 1983;15:345-9. (In Japanese.)

3 Aoki N. Acute toxic encephalopathy with symmetrical low density areas in the thalami and the cerebellum. Childs Nerv Syst 1985;1:62-5.

4 Mizuguchi M, Kamoshita S. Neuropathology of Reye syndrome. Fournal of Pediatric Practice 1986;49:1027-35. (In Japanese.)

5 Kumagai K, Goto K, Obata J, et al. Serial CT findings of acute encephalopathy. In: Yamashita F, ed. 1983 Annual report of the diseases of unknown etiology in infancy research report of the diseases of unknown etiology in infancy research Welfare of Japan, 1984:26-30. (In Japanese.)

6 Inoue M, Sato K, Nomura T, et al. A case of Reye syndrome accompanying influenza $B$ virus infection with CT findings suggestive of Leigh's encephalopathy fournal of the Fapanese Pediatric Society 1984;88 1429-35. (In Japanese)

7 Hino T, Sai H, Morikawa Y, Mizuta R, Okuno T. A case of clinical Reye syndrome presenting characteristic CT changes. No To Hattatsu 1984;16:210-7. (In Japanese)

8 Fukuyama Y, Awaya Y. Serial CT findings of acute encephalopathy with reference to the cases showing bithalamic low density areas. In: Yamashita F, ed. 1984 Annual report of diseases of unknown etiology in infancy research committee. Vol. 1, Tokyo: The Ministry of Health and Welfare of Japan, 1985:165-70. (In Japanese.)

9 Oda Y, Sato M, Ishizuka T, Yamazaki A. Two cases of Reye syndrome following exanthema subitum. fapanese fournal of Pediatrics 1987;40:2967-72. (In Japanese.)

10 Maeda K, Abe Y, Sasamoto A, et al. Three cases of acute encephalopathy with low density in thalamic regions on CT. Fapanese fournal of Pediatrics 1987;40:99-104. (In Japanese.)
11 Sasamoto A, Saitou S, Tateno A, et al. A case of acute encephalopathy with low density in bilateral thalamus. Pediatrics Fapan 1988;29:557-62. (In Japanese.)

12 Tateno A, Sakai K, Sakai S, Koya N, Aoki T. Computed tomography of bilateral thalamic hypodensity in acute encephalopathy. $\mathcal{F}$ Comput Assist Tomogr 1988;12:637-9.

13 Kiyonaga T, Kouno T, Shimoda K, Narumi F, Miyazaki $M$, Kinoshita T. A case of acute encephalopathy presenting characteristic CT changes. Fapanese fournal of Pediatrics 1988;41:92-6. (In Japanese.)

14 Waki K, Kawamura M, Kidani K, Ishigame $\mathrm{K}$. A case of acute encephalopathy with hypodensity of bilateral thalamus and cerebellum. fapanese fournal of Pediatrics mus and cerebellum. Fapanese

15 Momota K, Takesima Y, Maehara K, et al. A case of acute encephalopathy with hypodensity of bilateral thalamus, cerebellum and periventricular region on cranial CT [abstract]. No To Hattatsu 1989;21 (suppl):270. (In Japanese.)

16 Tanaka K, Kitoh Y, Okumura T, Tagi J, Nakasako H, Uemura $M$. Four cases of encephalopathy with bilateral symmetrical hypodensity on cranial CT [abstract]. No To Hattatsu 1989;21 (suppl): 270 (In Japanese.)

17 Yanagawa T, Minami Y, Kusumoto S, et al. Six cases of acute encephalopathy [abstract]. No To Hattatsu 1989;21 (suppl): 271. (In Japanese.)

18 Sunaga $Y$, Fujinaga T, Tamura H. A study on computed tomography of three cases of Reye syndrome. No To Hattatsu 1991;23:100-2. (In Japanese.)

19 Takahashi N, Nakano S, Matsuda S, et al. A case of acute encephalopathy presenting unusual cranial CT findings [abstract]. Fournal of the Fapanese Pediatric Society 1991;95.1022. (In Japanese.)

20 Iai $M$, Tanabe $Y$, Goto $M$. A case of acute encephalopathy with symmetrical low density areas in the thalami on $\mathrm{CT}$; serial CT and MRI findings. No To Hattatsu 1992;24:370-4. (In Japanese.)

21 Nagai T, Yagishita A, Tsuchiya Y, Asamura S, Kurokawa $\mathrm{H}$, Matsuo N. Symmetrical thalamic lesions on CT in influenza $A$ virus infection presenting with and without Reye syndrome. Brain Dev 1993;15:67-74.

22 Miyajima T, Hoshika A, Ogiwara M, et al. A study on two cases of acute encephalopathy with specific imaging findings and spindle coma-Association between $14 \mathrm{~Hz}$ spindle and lateral nucleus of the thalamus [abstract]. In: Proceedings of the 19th Meeting of Kanto In: Proceedings of the 19th Meeting of Kanto
Pediatric Neurology Society. Tokyo: 1992;16. (In Pediatric

23 Ushioda T. Acute encephalopathy with hypodensity in bilateral thalami on CT. Tama Neuroradiology Case Conference, 1992;9.

24 Kitamura K, Higuchi K, Ihara T, Kamiya H. A case of acute encephalopathy in which computed tomography revealed bilateral hypodensity of the thalamus due to thrombosis of the straight sinus. Fournal of the fapanese Pediatricians Society 1993;97:1060-64. (In Japanese.)

25 Takano T, Matsui E, Yamano T, Shimada $M$, Okumura $\mathrm{K}$. A case of clinical Reye syndrome with symmetrical abnormal signal areas in the pons and thalami by MRI No To Hattatsu 1994;26:63-7. (In Japanese.)

26 Crisp DE, Sieger RL, Bale JF, Thompson JA. Hemorrhagic cerebral infarction in the hemolytic-uremic syndrome. F Pediatr 1981;99:273-6.

27 Vles JSH, de Vries LS, Wilms G, de Roo M, Casaer PJM. Computed cranial tomography, magnetic resonance imaging and single photon emission computed tomography in hemorrhagic shock and encephalopathy syndrome: A report of three cases. Neuropediatrics 1992;23:24-7.

28 Corey L, Rubin RJ, Bregman D, Gregg MB. Diagnostic criteria for influenza B-associated Reye's syndrome: clinical vs. pathologic criteria. Pediatrics $1977 ; 60: 702-8$.

29 Cullity GJ, Kakulas BA. Encephalopathy and fatty degeneration of the viscera: An evaluation Brain 1970;93:77-88.

30 Partin JC, Schubert WK, Partin JS. Mitochondrial ultrastructure in Reye's syndrome (encephalopathy and fatty degeneration of the viscera). $N$ Engl f Med 1971;285: 1339-43.

31 Friede RL. Developmental neuropathology, 2nd ed. Berlin: Springer-Verlag, 1989.

32 Goutieres F, Aicardi J. Acute neurological dysfunction associated with destructive lesions of the basal ganglia in children. Ann Neurol 1982;12:328-32.

33 Adams RD, Kubik CS. The morbid anatomy of the demyelinative diseases. Am f Med 1952;12:510-46.

34 Evans H, Bourgeois CH, Comer DS, Keschamras N. Brain lesions in Reye's syndrome. Arch Pathol 1970;90 543-6.

35 Okuno T. Computed tomography in pediatric neurological diseases: with emphasis on CT of the patients with movement disturbances. No To Hattatsu 1979;11: 116-22. (In Japanese.)

36 Kalbag RM, Woolf AL. Thrombosis and thrombophlebitis of cerebral veins and dural sinuses. In: Vinken PJ, Bruyn $\mathrm{GW}$, eds. Handbook of clinical neurology. Vascular diseases of the nervous system. Vol 12. Amsterdam: Northof the nervous system.

37 Barkovich AJ, Kjos BO, Jackson DE, Norman D. Normal maturation of the neonatal period and infant brain: MR maturation of the neonatal period and infant $\mathrm{b}$

38 Mizuguchi $M$, Tomonaga $M$, Fukusato $T$, Asano $M$ Acute necrotizing encephalopathy with widespread edematous lesions of symmetrical distribution. Acta Neuropathol 1989;78:108-11. 